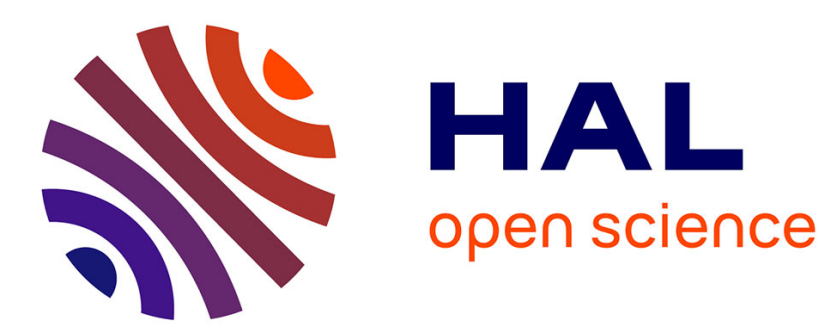

\title{
Statistical damage localization with stochastic load vectors using multiple mode sets
}

Md Delwar Hossain Bhuyan, Michael Döhler, Yann Lecieux, Laurent Mevel, Franck Schoefs

\section{- To cite this version:}

Md Delwar Hossain Bhuyan, Michael Döhler, Yann Lecieux, Laurent Mevel, Franck Schoefs. Statistical damage localization with stochastic load vectors using multiple mode sets. Structural Health Monitoring, 2017, 16 (5), pp.518-535. 10.1177/1475921717714447 . hal-01577250

\section{HAL Id: hal-01577250 \\ https://hal.inria.fr/hal-01577250}

Submitted on 25 Aug 2017

HAL is a multi-disciplinary open access archive for the deposit and dissemination of scientific research documents, whether they are published or not. The documents may come from teaching and research institutions in France or abroad, or from public or private research centers.
L'archive ouverte pluridisciplinaire HAL, est destinée au dépôt et à la diffusion de documents scientifiques de niveau recherche, publiés ou non, émanant des établissements d'enseignement et de recherche français ou étrangers, des laboratoires publics ou privés. 


\title{
Statistical damage localization with stochastic load vectors using multiple mode sets
}

\author{
Md Delwar Hossain Bhuyan ${ }^{1}$, Michael Döhler ${ }^{1}$, Yann Lecieux ${ }^{2}$, Laurent Mevel ${ }^{1}$ and Franck \\ Schoefs $^{2}$
}

\begin{abstract}
The Stochastic Dynamic Damage Locating Vector (SDDLV) method is an output-only damage localization method based on both a Finite Element (FE) model of the structure and modal parameters estimated from output-only measurements in the damage and reference states of the system. A vector is obtained in the null space of the changes in the transfer matrix from both states and then applied as a load vector to the model. The damage location is related to this stress where it is close to zero. In previous works, an important theoretical limitation was that the number of modes used in the computation related to the transfer function could not be higher than the number of sensors located on the structure. It would be nonetheless desirable not to discard information from the identification procedure. In this paper, the SDDLV method has been extended with a joint statistical approach for multiple mode sets, overcoming this restriction on the number of modes. Another problem is that the performance of the method can change considerably depending of the Laplace variable where the transfer function is evaluated. Particular attention is given to this choice and how to optimize it. The new approach is validated in numerical simulations and on experimental data, where the outcomes for multiple mode sets are compared with only using a single mode set. From these results, it can be seen that the success rate of finding the correct damage localization is increased when using multiple mode sets instead of a single mode set.
\end{abstract}

\section{Keywords}

Damage localization, Load vectors, Covariance analysis, Hypothesis testing, Modal truncation

\section{Introduction}

Vibration-based structural health monitoring (SHM) techniques have been actively developed in the last decades ${ }^{1-3}$, for example for the monitoring of bridges, buildings or offshore structures. Physical changes in the structure due to damage induce changes in the modal characteristics of the structure, which can be monitored through output-only vibration measurements.

Monitoring-based structural diagnosis is usually divided into five subtasks of increasing difficulty ${ }^{4}$ : damage detection (level 1), damage localization (level 2), identification of damage type (level 3), quantification of the damage extent (level 4) and prediction of the remaining service life (level 5). When performing these tasks in a cascade fashion, the full structural diagnosis problem is amenable to a solution. Methods for damage detection have reached some maturity so far, e.g. with data-driven algorithms adapted from pattern classification and statistical process control ${ }^{1 ; 5-9}$.

While damage detection can operate purely data-driven, by comparing a current dataset to a reference, damage localization requires some link between the data and the physical properties of the structure, which is often given by a finite element (FE) model of the structure or by directly assuming specific structural types like beams, plates or rotating machinery in their derivation. Data-driven damage localization methods ${ }^{2 ; 3}$ are usually designed for particular structural types, often in combination with dense sensor grids, and are not easily generalizable to arbitrary structural types. Model-based methods update the parameters of an FE model of the healthy structure based on measurements from the damaged system, and damage is located in the regions of the model where parameters are modified ${ }^{10 ; 11}$. While this approach is applicable to arbitrary structural types, it is often too poorly conditioned to be successful in practice due to the huge FE parameter dimension in comparison to relatively few modal parameters that can be extracted from data, and often user interaction is required by an experienced engineer in the updating process.

Alternative damage localization methods with a theoretical background combine properties of the data-driven and model-based approaches. They are based on data-driven features from measurements of the reference and damaged states, which are confronted to a FE model of the investigated structure to define damage indicators for the elements of the FE model, without updating its parameters ${ }^{12}$. For example, localization can be performed by statistical tests on a parametrized residual vector that is computed from measurements ${ }^{13 ; 14}$, or by interrogating changes in the flexibility of

\footnotetext{
${ }^{1}$ Inria/IFSTTAR, I4S team, France
}

${ }^{2}$ GeM, Université de Nantes, France

\section{Corresponding author:}

Michael Döhler, Inria/IFSTTAR, I4S team, Campus de Beaulieu, 35042 Rennes, France.

Email: michael.doehler@inria.fr 
a structure ${ }^{15 ; 16}$ and extracting localization information based on the FE model.

Belonging to the latter category, the Stochastic Dynamic Damage Locating Vector (SDDLV) approach ${ }^{16}$ is a vibration-based damage localization technique using both finite element information and modal parameters estimated from output data. Damage is assumed to be related to stiffness loss, which is coherent with common damage models ${ }^{17 ; 18}$. From the modal estimates in both reference and damaged states, a vector in the null space of the difference between the respective transfer matrices is obtained at the sensor positions for some Laplace variable $s$ in the complex plane. It has been shown that when applying this load vector to the FE model of the healthy structure, then the resulting stress field is zero at the damaged element. Since the estimates of the modal parameters are naturally subject to variance errors ${ }^{19-24}$, the resulting stress estimate is not exactly zero at the damaged element, but only close to zero. Based on that uncertainty information, a statistical extension of the SDDLV method was developed in ${ }^{25 ; 26}$ for deciding if an element is damaged.

In previous works on the SDDLV ${ }^{16 ; 25-27}$, the number of sensors needed to be at least as high as the number of identified modes. This is a restriction when more modes are identified from the measurements than the available number of sensors, and not all the available information could be taken into account. At the same time, it is desirable to perform damage localization with a low number of sensors. In this paper, the SDDLV method is developed with a joint statistical evaluation using multiple mode sets, overcoming the limitation on the number of sensors and taking into account all identified modal information. It is demonstrated that the computation of stress for multiple mode sets increases the information content about the damaged or nondamaged elements of the structure with respect to a limited number of modes. Finally, all stress values corresponding to each element are tested for damage in a statistical hypothesis test where the computed stresses are evaluated with their joint covariance.

Assessing the performance of the method is a requirement for showing the benefits of this approach. A proper criterion for the evaluation of the success rate is proposed based on Monte Carlo simulations. The performance of the SDDLV approach is strongly dependent on the choice of the Laplace variable $s$ where the transfer function is evaluated due to the different influence of modal truncation errors. Accommodating multiple $s$-values has been treated in ${ }^{26}$. Still, the choice of the Laplace variable is a complicated part of the procedure, even if past guidelines suggest choosing this variable around the identified modes in the complex plane to reduce modal truncation errors. This motivates the performance analysis of the method in Monte Carlo simulations for many $s$-variables in the complex plane in this paper, where the success rate of correct damage localization is evaluated in dependence of the $s$-values. The performance of the proposed approach is evaluated under the requirement that the particular choice of $s$ should not be critical. Indeed, the simulation results show that the effect of $s$-values with poor performance is mitigated when treating all available modes with the statistical multiple mode set strategy of this paper. This leads to significant improvement of the localization success rate with the proposed method and less dependence on the particular choice of the $s$-values.

This paper is organized as follows. First, the SDDLV method is presented and the removal of the limiting restriction on the number of modes is discussed using multiple mode sets. Then, the statistical damage localization approach is derived using these multiple mode sets. Finally, the new approach is applied on numerical simulations and experimental data to evaluate the performance of the approach, and conclusions of the work are given.

\section{Damage localization approach (SDDLV)}

The Stochastic Dynamic Damage Locating Vector (SDDLV) approach is an output-only damage localization method based on interrogating changes $\delta G(s)$ in the transfer matrix $G(s)$ of a system in both reference and damaged states $^{16}$, where $s$ is a Laplace variable in the complex plane. A vector is obtained in the null space of $\delta G(s)$ from system identification results using output-only measurements corresponding to both states. Then this load vector is applied to the Finite Element (FE) model of the structure for the computation of a stress field over the structure. The damage is located where the computed stress is zero or close to zero in practice ${ }^{15 ; 16}$. In this section, the deterministic computation of the stress field for damage localization is summarized.

\section{Modeling}

We assume that the behavior of a mechanical structure can be described by a linear time-invariant (LTI) dynamic system

$$
M \ddot{\mathcal{X}}(t)+C \dot{\mathcal{X}}(t)+K \mathcal{X}(t)=f(t)
$$

where $M, C, K \in \mathbb{R}^{d \times d}$ are the mass, damping and stiffness matrices, respectively, $t$ indicates continuous time and $\mathcal{X} \in$ $\mathbb{R}^{d}$ denotes the displacements at the $d$ degrees of freedom (DOF) of the structure. The external force $f(t)$ is not measurable and modeled as white noise.

Let the dynamic system (1) be observed at $r$ coordinates, where often $r \ll d$ in practice. Since $f(t)$ is unmeasured, it can be substituted with a fictive force $e(t) \in \mathbb{R}^{r}$ acting only in the measured coordinates and that regenerates the measured output. Furthermore, defining $x=\left[\begin{array}{ll}\mathcal{X}^{T} & \dot{\mathcal{X}}^{T}\end{array}\right]^{T}$, this leads to the corresponding continuous-time state-space model

$$
\left\{\begin{array}{l}
\dot{x}(t)=A_{c} x(t)+B_{c} e(t) \\
y(t)=C_{c} x(t)+D_{c} e(t)
\end{array}\right.
$$

with state vector $x \in \mathbb{R}^{n}$, output vector $y \in \mathbb{R}^{r}$, the state transition matrix $A_{c} \in \mathbb{R}^{n \times n}$ and output matrix $C_{c} \in \mathbb{R}^{r \times n}$, where $n=2 d$ is the system order and $r$ is the number of outputs. Since the input of the system is replaced by the fictive force $e \in \mathbb{R}^{r}$, the input influence matrix and direct transmission matrix are of size $B_{c} \in \mathbb{R}^{n \times r}$ and $D_{c} \in \mathbb{R}^{r \times r}$ respectively. However, only the system matrices $A_{c}$ and $C_{c}$ are relevant from output-only system identification, and the non-identified matrices $B_{c}$ and $D_{c}$ will only be needed in the derivation of estimates related to the transfer matrix. From Stochastic Subspace Identification (SSI) ${ }^{28-30}$, modal parameter estimates and subsequently the estimates $\widehat{A}_{c}$ and $\widehat{C}_{c}$ can be obtained from output only measurements, details are given in Appendix A. 
Note that environmental variability, e.g. due to temperature changes, may affect the structural properties or boundary conditions and thus the modal parameter estimates ${ }^{31}$. These effects are not taken into account in the presented models. Since the considered damage localization approach operates on the modal parameter estimates, and environmental variability can be removed from these estimates in a preprocessing step with diverse methods ${ }^{5 ; 32}$, we do not consider this problem further in this paper.

\section{Computation of damage indicator}

The damage indicator is based on the transfer matrix difference $\delta G(s)$ between reference and damaged states. However, the transfer matrix

$$
G(s)=C_{c}\left(s I-A_{c}\right)^{-1} B_{c} \in \mathbb{C}^{r \times r}
$$

itself cannot be estimated from output-only measurements since matrices $B_{c}$ and $D_{c}$ cannot be estimated. Matrix $B_{c}$ can be replaced by using the relationships ${ }^{16 ; 27}$

$$
\begin{aligned}
C_{c} B_{c} & =0 \\
C_{c} A_{c} B_{c} & =D_{c}
\end{aligned}
$$

and formulating the least-squares problem

$$
\left[\begin{array}{c}
C_{c} A_{c} \\
C_{c}
\end{array}\right] B_{c}=\left[\begin{array}{l}
I \\
0
\end{array}\right] D_{c}
$$

which has a solution for $B_{c}$ under the condition that the system order satisfies $n \leq 2 r$, i.e. the number $m$ of identified modes satisfies $m \leq r$. Then, ${ }^{16 ; 27}$

$$
G(s)=R(s) D_{c},
$$

where

$$
R(s)=C_{c}\left(s I-A_{c}\right)^{-1}\left[\begin{array}{c}
C_{c} A_{c} \\
C_{c}
\end{array}\right]^{\dagger}\left[\begin{array}{l}
I \\
0
\end{array}\right] .
$$

In (3), $I$ is the identity matrix of size $r \times r, 0$ is the zero matrix of size $r \times r$, and ${ }^{\dagger}$ denotes the Moore-Penrose pseudoinverse. The difference between the transfer matrices in both damaged (variables with tilde) and healthy states is $\delta G(s)=\tilde{G}(s)-G(s)$. Assume that damage is due to changes in stiffness and mass is constant. Then $D_{c}=\tilde{D}_{c}$, and the matrices $\delta G(s)$ and $\delta R(s)^{T}=\tilde{R}(s)^{T}-R(s)^{T}$ have the same null space ${ }^{16}$. The desired load vector $v(s)$ is obtained from the null space of the $\delta R(s)^{T}$ from Singular Value Decomposition (SVD)

$$
\delta R(s)^{T}=U \Sigma V^{H}=\left[\begin{array}{ll}
U_{1} & U_{2}
\end{array}\right]\left[\begin{array}{cc}
\Sigma_{1} & 0 \\
0 & \Sigma_{2}
\end{array}\right]\left[\begin{array}{ll}
V_{1} & V_{2}
\end{array}\right]^{H},
$$

where $U, \Sigma, V \in \mathbb{C}^{r \times r}, \quad \Sigma_{2} \approx 0$ and $H$ indicates the conjugate transpose. Let $n_{\mathrm{im}}$ be the dimension of the image $U_{1}$ and $\left(r-n_{\mathrm{im}}\right)$ be the dimension of the null space $V_{2}$, where $n_{\text {im }}$ depends on the kind and number of damaged elements ${ }^{15}$. The load vector $v(s)$ is chosen from the null space $V_{2}$, e.g. as the last column of $V_{2}$. Note that only output data is necessary for its computation. To compute the stress field over the elements of the structure, the load vector $v(s)$ is applied to the FE model of the structure.
First, the vector $v(s)$ is expanded to load vector $\nu(s)$ at all DOFs of the model, whose entries are those of $v(s)$ at the sensor coordinates and zero elsewhere. From this vector, the nodal displacements $y(s)=\left(M s^{2}+C s+K\right)^{-1} \nu(s)$ are computed based on the FE model at all DOFs, from which stress resultants are evaluated for each structural element of the model and stacked into stress vector $S(s)$. This relation between stress and load is linear and can be expressed by a matrix $\mathcal{L}_{\text {model }}(s) \in \mathbb{C}^{l \times r}$ based on the FE model of the structure $^{16 ; 25}$, satisfying

$$
S(s)=\mathcal{L}_{\text {model }}(s) v(s) .
$$

The stress vector $S(s)$ indicates potential damage for elements with corresponding entries in $S(s)$ that are close to zero ${ }^{15 ; 16}$. When estimated, these stresses are not exactly zero but small in practice because of modal truncation, model errors and variance errors from measurements.

\section{Stress aggregation for robustness}

Due to truncation and model errors, it is recommended to compute the load vector $v(s)$ and the resulting stress $S(s)$ for several $s$-values $s_{w}, w=1, \ldots, \kappa$, and to aggregate results. To minimize error, the $s$-values should be chosen in the vicinity of the identified poles of the system but not too close to them ${ }^{16 ; 26}$. After identification of the system matrices in both states, the computations of (4)-(5) are repeated for each $s_{w}$ to get the respective stress vectors $S\left(s_{w}\right)$. For multiple $s$ values, a deterministic stress aggregation is obtained for each element $t$ as ${ }^{16}$

$$
\bar{S}_{t}=\sum_{w=1}^{\kappa}\left|S_{t}\left(s_{w}\right)\right|
$$

In previous works ${ }^{26}$, this deterministic aggregation has been replaced by a statistical aggregation, where the intrinsic uncertainty of the stress estimation from finite measurement data is taken into account.

In the following, we address the restriction on the number of modes in the computation of the load vector by using multiple mode sets. The resulting stress from each mode set will be statistically aggregated for damage localization.

\section{Multiple mode sets for SDDLV}

In practice, there may be more modes available from identification than number of sensors on the structure. It will be meaningful to utilize this information completely from the identification procedure. In the SDDLV ${ }^{16}$, it was not possible to use all modes in this case for the stress computation due to the theoretical restriction $n \leq 2 r$, where $n=2 m$ is the system order and $r$ is the number of sensors. Note that $m$ is the number of conjugated complex mode pairs identified from datasets where $m$ has to satisfy the constraint $m \leq r$.

This constraint is due to the fact that system matrix $B_{c}$ in (2) cannot be estimated from output-only measurements for the computation of the transfer matrix. Under this constraint, expression (3) is available, which allows the computation of the load vector in the null space of $\delta G(s)$ from output-only measurements.

In order to remove the restriction $m \leq r$ on the number of modes, the computation of stress from different mode sets is investigated in the following, where the current 
restriction is satisfied for the number of modes in each mode set. This allows considering more than $r$ modes in the analysis. By taking into account more of the identified modes, it is expected that the information content for damage localization should increase. Load vectors and the respective stress are computed for each mode set, using one or several $s$-values.

Let the identified modes of the system be split into $n_{s}$ mode sets $\mathcal{M}_{j}, j=1, \ldots, n_{s}$, containing $m_{j}$ modes each, where the condition $m_{j} \leq r$ is satisfied for each mode set. Then, the new method of this paper takes into account the identified data from all mode sets as follows.

The modal parameters of the structure are identified from SSI using measurement data of the healthy and of the damaged state. From the modal parameters corresponding to each mode set $\mathcal{M}_{j}$, the system matrices $A_{c}^{j}$ and $C_{c}^{j}$ are assembled in the healthy and damaged states as detailed in Appendix A in (22). Then, the computation of the load vector $v^{j}(s)$ is carried out separately for each mode set, i.e. the "transfer matrix" resultant $R^{j}(s)$ is computed from both states as in (3) and the load vector $v^{j}(s)$ is obtained in the null space of $R^{j}(s)$ from the SVD as in (4). The stress vectors $S^{j}(s)=\mathcal{L}_{\text {model }}(s) v^{j}(s)$ are computed for each mode set and $s$-value, together with their uncertainty. Finally, damage localization is performed based on a joint statistical evaluation of the computed stresses.

\section{Statistical evaluation for SDDLV using multiple mode sets}

For the damage localization algorithm, estimates of the modal parameters are obtained in the damaged and undamaged states using SSI ${ }^{26}$. For each mode set, they are the starting point of the computations of the load vectors and associated stresses for damage localization. Their identification is subject to variance errors because of unknown excitation, measurement noise and limited data length. The uncertainties in the estimates are penalizing the quality and precision of the damage localization results. For making decisions about damaged elements of the structure, these uncertainties need to be taken into account to decide whether stress of an element is significantly close to zero or not. In previous works ${ }^{25 ; 26}$, the uncertainty of the stress vector $S(s)$ in (5) was quantified for a single mode set at one or several $s$-values. In this section, the uncertainty computation of the stress vector $S^{j}(s)$ is derived for different mode sets $\mathcal{M}_{j}, j=1, \ldots, n_{s}$, for any choice of $s$, and the joint evaluation of the different stress results is described for each structural element in a statistical test.

The modal parameters are obtained from covariancedriven subspace identification (cov-SSI) ${ }^{22 ; 29 ; 30}$. They are estimated from measurement data through the computation of the Hankel matrix $\mathcal{H}$ containing the estimated output covariances of the system (see details in Appendix A). Let $\Sigma_{\mathcal{H}}$ be the covariance of $\operatorname{vec}(\mathcal{H})$, where $\operatorname{vec}(\cdot)$ defines the column stacking vectorization operator. This covariance can easily be estimated from the measurement data ${ }^{20 ; 22}$, details are given in Appendix B. Then, the covariance of a vectorvalued function $g(\mathcal{H})$ can be approximated by

$$
\operatorname{cov}(g(\mathcal{H})) \approx \mathcal{J}_{g, \mathcal{H}} \Sigma_{\mathcal{H}} \mathcal{J}_{g, \mathcal{H}}^{T},
$$

where $\mathcal{J}_{g, \mathcal{H}}=\partial g(\mathcal{H}) / \partial \operatorname{vec}(\mathcal{H})$ is the sensitivity of the function $g$. Since the output covariances and the Hankel matrix are asymptotically Gaussian variables ${ }^{33}$ (when number of measurements is large), the statistical delta method $^{34}$ ensures that expression (7) is asymptotically exact. The required sensitivity can be obtained analytically through a first-order perturbation $\triangle g$ of the function $g$, which yields

$$
\triangle g=\mathcal{J}_{g, \mathcal{H}} \operatorname{vec}(\triangle \mathcal{H})
$$

With this strategy, the covariance $\Sigma_{\mathcal{H}}$ of an estimate of Hankel matrix $\mathcal{H}$ can be propagated to any function of $\mathcal{H}$, particularly to the modal parameters and to the stress estimate $S(s)$.

Note that some of the matrices and vectors in the derivation of the damage localization approach are complexvalued variables. To deal with their uncertainties, define an equivalent real-valued notation for any matrix $Q$ as

$$
Q_{\mathrm{Re}} \stackrel{\text { def }}{=}\left[\begin{array}{cc}
\Re(Q) & -\Im(Q) \\
\Im(Q) & \Re(Q)
\end{array}\right], Q_{\mathrm{re}} \stackrel{\text { def }}{=}\left[\begin{array}{l}
\Re(Q) \\
\Im(Q)
\end{array}\right],
$$

where $\Re(\cdot)$ and $\Im(\cdot)$ denote the real and imaginary parts, respectively.

For multiple mode sets $\mathcal{M}_{j}, j=1, \ldots, n_{s}$, the sensitivities of the stress estimate $S^{j}(s)$ are derived with respect to estimates $A_{c}^{j}, C_{c}^{j}$ and subsequently to the Hankel matrix $\mathcal{H}$ in both the damaged and healthy states. Then, the covariance of $S^{j}(s)$ can be obtained as in (7).

\section{Sensitivity of the system matrices $A_{c}$ and $C_{c}$ from subspace identification}

In this section, the covariance of the system matrices $A_{c}^{j}$ and $C_{c}^{j}$ is computed for each mode set $\mathcal{M}_{j}$, starting from the identified eigenvalues $\lambda_{c, l}^{j}$ and mode shapes $\varphi_{l}^{j}, l=$ $1, \ldots, m_{j}$, for each mode set. Based on the SSI approach, their perturbation is linked to the Hankel matrix by

$$
\begin{aligned}
\triangle \lambda_{c, l}^{j} & =\mathcal{J}_{\lambda_{c, l}^{j}, \mathcal{H}} \operatorname{vec}(\triangle \mathcal{H}), \\
\triangle \varphi_{l}^{j} & =\mathcal{J}_{\varphi_{l}^{j}, \mathcal{H}} \operatorname{vec}(\triangle \mathcal{H})
\end{aligned}
$$

where the sensitivity matrices $\mathcal{J}_{\lambda_{c, l}^{j}, \mathcal{H}} \in \mathbb{C}^{1 \times h}$ and $\mathcal{J}_{\varphi_{l}^{j}, \mathcal{H}} \in$ $\mathbb{C}^{r \times h}$ with $h=\operatorname{dim}(\operatorname{vec}(\mathcal{H}))$ are derived in ${ }^{20 ; 22}$. The system matrices $A_{c}^{j}$ and $C_{c}^{j}$ are assembled from the eigenvalues and mode shapes for each mode set $\mathcal{M}_{j}$ as detailed in Appendix A. The sensitivities of the vectorized system matrices yield accordingly

$$
\begin{aligned}
\operatorname{vec}\left(\triangle A_{c}^{j}\right) & =\mathcal{J}_{A_{c}, \mathcal{H}}^{j} \operatorname{vec}(\triangle \mathcal{H}), \\
\operatorname{vec}\left(\triangle C_{c}^{j}\right) & =\mathcal{J}_{C_{c}, \mathcal{H}^{j}}^{j} \operatorname{vec}(\triangle \mathcal{H}),
\end{aligned}
$$

where the sensitivities $\mathcal{J}_{A_{c}, \mathcal{H}}^{j}$ and $\mathcal{J}_{C_{c}, \mathcal{H}}^{j}$ are directly obtained from the eigenvalue and mode shape sensitivities $\mathcal{J}_{\lambda_{c, l}^{j}, \mathcal{H}}$ and $\mathcal{J}_{\varphi_{l}^{j}, \mathcal{H}}$ as detailed in ${ }^{26}$.

\section{Sensitivity of the stress vector $S^{j}(s)$}

Now, the uncertainty of the system matrices is propagated to matrix $R^{j}(s)$ in (3) for both healthy and damaged states, then to the load vector in the null space of $\delta R^{j}(s)^{T}=\tilde{R}^{j}(s)^{T}-$ 
$R^{j}(s)^{T}$ and finally to the stress vector $S^{j}(s)$. It holds that

$$
\begin{aligned}
\operatorname{vec}\left(\triangle R^{j}(s)^{T}\right)_{\mathrm{re}} & =\mathcal{J}_{R(s)^{T},\left(A_{c}, C_{c}\right)}^{j}\left[\begin{array}{c}
\operatorname{vec}\left(\triangle A_{c}^{j}\right) \\
\operatorname{vec}\left(\triangle C_{c}^{j}\right)
\end{array}\right] \\
\triangle v^{j}(s)_{\mathrm{re}} & =\mathcal{J}_{v(s), \delta R(s)^{T}}^{j}\left(\operatorname{vec}\left(\triangle \delta R^{j}(s)^{T}\right)\right)_{\mathrm{re}} \\
\triangle S^{j}(s)_{\mathrm{re}} & =\left(\mathcal{L}_{\text {model }}(s)\right)_{\mathrm{Re}} \triangle v^{j}(s)_{\mathrm{re}}
\end{aligned}
$$

where the respective sensitivity matrices have been derived in detail in ${ }^{26}$. For a joint evaluation of the stress for multiple mode sets, the uncertainty of the stress needs to be related to a common factor, which is the uncertainty of the Hankel matrix. Combining (9) and (10), it holds

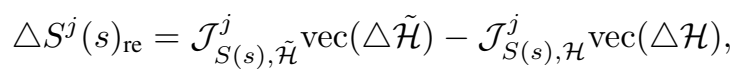

where

$$
\begin{gathered}
\mathcal{J}_{S(s), \mathcal{H}}^{j}=\left(\mathcal{L}_{\text {model }}(s)\right)_{\operatorname{Re}} \mathcal{J}_{v(s), \delta R(s)^{T}}^{j} \\
\cdot \mathcal{J}_{R(s)^{T},\left(A_{c}, C_{c}\right)}^{j}\left[\begin{array}{c}
\mathcal{J}_{A_{c}, \mathcal{H}}^{j} \\
\mathcal{J}_{C_{c}, \mathcal{H}}^{j}
\end{array}\right], \\
\mathcal{J}_{S(s), \tilde{\mathcal{H}}}^{j}=\left(\mathcal{L}_{\text {model }}(s)\right)_{\operatorname{Re}} \mathcal{J}_{v(s), \delta R(s)^{T}}^{j} \\
\cdot \mathcal{J}_{\tilde{R}(s)^{T},\left(\tilde{A}_{c}, \tilde{C}_{c}\right)}^{j}\left[\begin{array}{c}
\mathcal{J}_{\tilde{A}_{c}, \tilde{\mathcal{H}}}^{j} \\
\mathcal{J}_{\tilde{C}_{c}, \tilde{\mathcal{H}}}^{j}
\end{array}\right] .
\end{gathered}
$$

Assume that the stress vector $S^{j}(s)$ is evaluated at a possibly different $s$-value $s=s^{j}$ for each mode set $\mathcal{M}_{j}$, $j=1, \ldots, n_{s}$. After stacking the real and imaginary parts of the stress vectors, the total stress vector is derived as

$$
S \stackrel{\text { def }}{=}\left[\begin{array}{c}
S^{1}\left(s^{1}\right)_{\mathrm{re}} \\
\vdots \\
S^{n_{s}}\left(s^{n_{s}}\right)_{\mathrm{re}}
\end{array}\right]
$$

and its uncertainty follows from (11) as

$$
\triangle S=\left[\begin{array}{c}
\mathcal{J}_{S\left(s^{1}\right), \tilde{\mathcal{H}}}^{1} \\
\vdots \\
\mathcal{J}_{S\left(s^{n_{s}}\right), \tilde{\mathcal{H}}}^{n_{s}}
\end{array}\right] \operatorname{vec}(\triangle \tilde{\mathcal{H}})-\left[\begin{array}{c}
\mathcal{J}_{S\left(s^{1}\right), \mathcal{H}}^{1} \\
\vdots \\
\mathcal{J}_{S\left(s^{n_{s}}\right), \mathcal{H}}^{n_{s}}
\end{array}\right] \operatorname{vec}(\triangle \mathcal{H})
$$

Note that vector $S$ contains the stress information for all mode sets at all elements of the structure.

\section{Joint statistical evaluation of stress}

Following from (7) and (13), the covariance $\Sigma_{S} \stackrel{\text { def }}{=}$ $\operatorname{cov}(\operatorname{vec}(S))$ of the total stress yields

$$
\begin{aligned}
\Sigma_{S}=\left[\begin{array}{c}
\mathcal{J}_{S\left(s^{1}\right), \tilde{\mathcal{H}}}^{1} \\
\vdots \\
\mathcal{J}_{S\left(s^{n_{s}}\right), \tilde{\mathcal{H}}}^{n_{s}}
\end{array}\right] \Sigma_{\tilde{\mathcal{H}}}\left[\begin{array}{c}
\mathcal{J}_{S\left(s^{1}\right), \tilde{\mathcal{H}}}^{1} \\
\vdots \\
\mathcal{J}_{S\left(s^{n_{s}}\right), \tilde{\mathcal{H}}}^{n_{s}}
\end{array}\right]^{T} \\
+\left[\begin{array}{c}
\mathcal{J}_{S\left(s^{1}\right), \mathcal{H}}^{1} \\
\vdots \\
\mathcal{J}_{S\left(s^{n_{s}}\right), \mathcal{H}}^{n_{s}}
\end{array}\right] \Sigma_{\mathcal{H}}\left[\begin{array}{c}
\mathcal{J}_{S\left(s^{1}\right), \mathcal{H}}^{1} \\
\vdots \\
\mathcal{J}_{S\left(s^{n_{s}}\right), \mathcal{H}}^{n_{s}}
\end{array}\right]^{T},
\end{aligned}
$$

since the datasets from reference and damaged states can be regarded as statistically independent. The covariance expression (14) leads to a new statistical approach for damage localization using multiple mode sets based on a statistical test for each element $t$ of the structure. In this approach, all stress components corresponding to a structural element $t$ are collected in a subvector $S_{t}$ of $S$, containing the information of all mode sets. Accordingly, the respective parts of the covariance matrix $\Sigma_{S}$ in (14) that correspond to element $t$ are collected in $\Sigma_{t}$, such that $\Sigma_{t}=\operatorname{cov}\left(S_{t}\right)$. Then, vector $S_{t}$ is tested for being zero in a hypothesis test. Since an estimate of the stress vector $S_{t}$ is asymptotically Gaussian distributed, a joint statistical evaluation of the computed stresses is derived in a $\chi^{2}$-test as

$$
\chi_{t}^{2}=S_{t}^{T} \Sigma_{t}^{-1} S_{t}
$$

for each structural element $t$. Since stress over damaged elements is zero in theory, potential damage is located in elements $t$ corresponding to the lowest values of $\chi_{t}^{2}$ among all elements.

\section{Joint stress evaluation for different s-values}

In the previous section, the computation of stress (12) was derived using only one Laplace variable for each mode set, while there is a possibility to use several Laplace variables. The computation of stress $S^{j}\left(s_{w}^{j}\right)$ can be easily generalized for several $s$-values for each mode set $\mathcal{M}_{j}$, denoted by $s_{w}^{j}$, where $w=1, \ldots, \kappa^{j}$ and $\kappa^{j}$ is the number of $s$-values used for mode set $\mathcal{M}_{j}$. After stacking the real and imaginary parts of the stress vectors for multiple $s$-values and mode sets, the joint stress vector writes analogously as

$$
S=\left[\begin{array}{c}
S^{1}\left(s_{1}^{1}\right)_{\mathrm{re}} \\
\vdots \\
S^{1}\left(s_{\kappa^{1}}^{1}\right)_{\mathrm{re}} \\
\vdots \\
S^{n_{s}}\left(s_{1}^{n_{s}}\right)_{\mathrm{re}} \\
\vdots \\
S^{n_{s}}\left(s_{\kappa^{n_{s}}}^{n^{n_{s}}}\right)_{\mathrm{re}}
\end{array}\right] .
$$

Then covariance of the stress (16) with respect to different $s$ values can be derived together with (14) for mode sets $\mathcal{M}_{j}$, $j=1, \ldots, n_{s}$, as

$$
\Sigma_{S}=\mathcal{J}_{S, \tilde{\mathcal{H}}} \Sigma_{\tilde{\mathcal{H}}} \mathcal{J}_{S, \tilde{\mathcal{H}}}^{T}+\mathcal{J}_{S, \mathcal{H}} \Sigma_{\mathcal{H}} \mathcal{J}_{S, \mathcal{H}}^{T}
$$

where

$$
\mathcal{J}_{S, \mathcal{H}}=\left[\begin{array}{c}
\mathcal{J}_{S\left(s_{1}^{1}\right), \mathcal{H}}^{1} \\
\vdots \\
\mathcal{J}_{S\left(s^{1}\right), \mathcal{H}}^{1} \\
\vdots \\
\mathcal{J}_{S\left(s_{1}^{n_{s}}\right), \mathcal{H}}^{n_{s}} \\
\vdots \\
\mathcal{J}_{S\left(s_{\kappa^{n_{s}}}^{n_{s}}\right), \mathcal{H}}^{n_{s}}
\end{array}\right], \quad \mathcal{J}_{S, \tilde{\mathcal{H}}}=\left[\begin{array}{c}
\mathcal{J}_{S\left(s_{1}^{1}\right), \tilde{\mathcal{H}}}^{1} \\
\vdots \\
\mathcal{J}_{S\left(s^{1}\right), \tilde{\mathcal{H}}}^{1} \\
\vdots \\
\mathcal{J}_{S\left(s_{1}^{n_{s}}\right), \tilde{\mathcal{H}}}^{n_{s}} \\
\vdots \\
\mathcal{J}_{S\left(s_{\kappa^{n_{s}}}^{n_{s}}\right), \tilde{\mathcal{H}}}^{n_{n}}
\end{array}\right] .
$$

Analogously to the previous section, the covariance expression (17) leads to a new statistical aggregation scheme 
for multiple mode sets using several $s$-values. For each structural element $t$, the corresponding stress subvector $S_{t}$ of $S$ in (16) is selected together with its covariance components $\Sigma_{t}$ of $\Sigma$ in (17). The stress vector $S_{t}$ is tested for being zero in a statistical hypothesis test as in (15) for each structural element $t$.

\section{Applications}

The damage localization method has been applied on numerical simulations and experimental data. A simple mass-spring chain and a more complex 3D cube beam model have been considered as numerical applications. These numerical applications are idealized test cases for the validation of the new developments of this paper, allowing in particular a statistical performance evaluation based on Monte-Carlo simulations. For simplicity, damage is assumed as stiffness loss in elements of a FE model. While no explicit link to a particular origin of such a damage is made, this is coherent with common damage models ${ }^{17 ; 18}$. Finally, the method has been applied to experimental data measured on a beam in a lab experiment.

For each application, the outcome of the damage localization results using multiple mode sets are compared with the ones using only one of the single mode sets separately. To obtain these results from simulated or measured data, the modes of the system are estimated using a stabilization diagram procedure with $\mathrm{SSI}^{30}$ in both reference and damaged states. They are estimated together with their covariance $^{22}$. Then, the system matrices and their covariance are assembled from the modes. For each single mode set, the stress vector and its uncertainty are computed. Finally, the estimated stress at the multiple (or single) mode sets and at multiple (or single) $s$-values is evaluated and statistically aggregated for each structural element with the new method of this paper. Comparisons to the deterministic aggregation are based on (6). Recall that stress values close to zero indicate potentially damaged elements.

\section{Performance evaluation of damage localization}

To analyze the performance of the proposed damage localization method using multiple mode sets, the success rate (or probability) of correct damage localization is evaluated for several sets of simulated measurement data. Each dataset is an independent realization and defines a Monte-Carlo experiment. In order to indicate if an element is potentially damaged or not, the $\chi_{t}^{2}$ value has to be computed for each structural element. For each Monte Carlo realization, damage localization is seen as successful when the lowest $\chi_{t}^{2}$ value among all elements is indeed at the damaged element. The success rate corresponds to the probability of detection or power of the test. Here, it is numerically obtained as the percentage of datasets, among all Monte Carlo experiments for which the $\chi_{t}^{2}$ value at the damaged element is the smallest $\chi_{t}^{2}$ value. The success rate depends on the chosen $s$-value(s) and serves as the performance indicator of the method when comparing it to localization procedures using only one mode set.

Note that the generation of several datasets allows the evaluation of the success rate, while in reality usually only one dataset is available.
In order to evaluate the influence of the $s$-values on the success rate of damage localization, each dataset in the Monte-Carlo simulations is evaluated for a set of $s$-values with different real and imaginary parts in order to obtain the success rate in dependence of $s$. The range of $s$-values has been chosen in the vicinity of the identified poles to reduce the effects of modal truncation in the transfer matrix estimates ${ }^{16}$. The resulting success rate as a function of $s$ is presented in 3D bar diagrams, where it is plotted on the $z$ axis in dependence of the real and imaginary parts of $s$ on the $x$ and $y$-axis.

Besides the presentation of the success rate of the statistical damage localization using several datasets from Monte-Carlo simulations, the statistical localization approach is compared to the underlying stress computation based on modal parameters from the model (theoretical stress) or from estimates (without statistical evaluation). The theoretical stress evaluation allows to assess the achievable localization accuracy under modal truncation, which is always present in practice. Comparing the localization results from stress estimation and its statistical evaluation allows to evaluate the importance of taking into account the statistical estimation errors in the new method.

\section{Numerical application 1: mass-spring chain}

In a first numerical application, the damage localization method has been applied on a mass-spring chain system with six DOFs as shown in Figure 1. The stiffness parameters are $k_{1}=k_{3}=k_{5}=4000, k_{2}=k_{4}=k_{6}=2000$, and the mass of all elements is 1 in suitable units. Damping is defined such that each mode has a damping ratio of $2 \%$. Damage is simulated by decreasing the stiffness of spring 4 by $10 \%$ of its original value. For damaged and undamaged states, the acceleration data length for each set is $N=50,000$. Data were generated from collocated white noise excitation using three sensors at elements 2, 4 and 6 in with sampling frequency of $50 \mathrm{~Hz}$, and white measurement noise with $5 \%$ magnitude of the outputs was added.

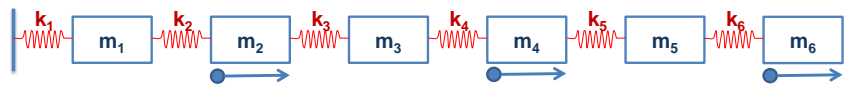

Figure 1. Mass-spring chain (with modal damping), three sensors.

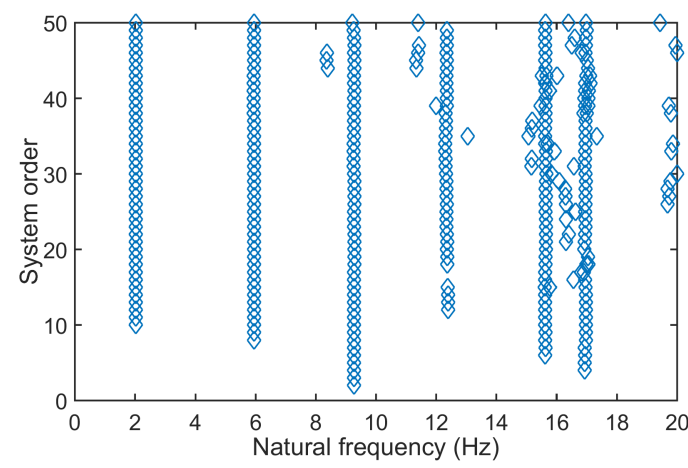

Figure 2. Stabilization diagram from mass-spring chain simulation in healthy state using SSI. 


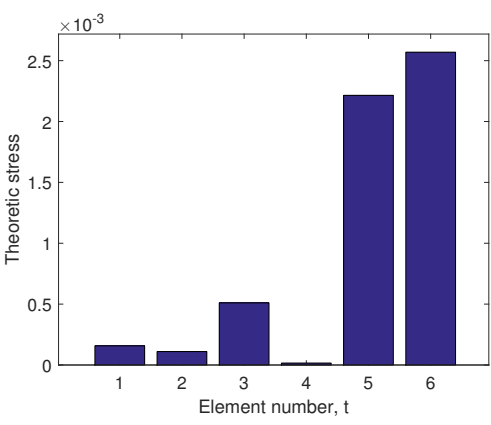

(a) Stress from exact modal parameters

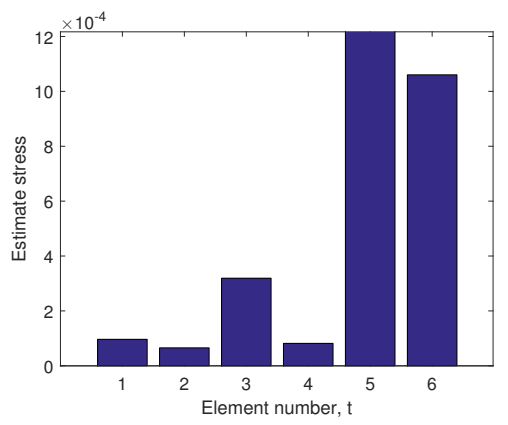

(b) Estimated stress from simulated dataset

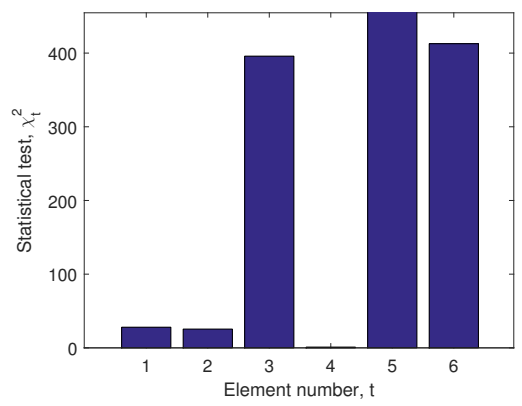

(c) Statistical evaluation in $\chi_{t}^{2}$-tests

Figure 3. Localization results at all elements of mass-spring chain using single mode set $\mathcal{M}_{1}$ using stress computation and statistical evaluation at $s=-2+51$ i. Damage is at element 4 .

All six modes of the structure (see Table 1) can be identified from the simulated measurements when using SSI. The respective stabilization diagram on a dataset from the healthy state is shown in Figure 2. Using three sensors in this example, only a limited set of three modes could be used for localization in previous works ${ }^{16 ; 25 ; 26}$ as the number of modes cannot be bigger than the number of sensors. For the proposed method in this paper, all modes can be considered by using two mode sets $\mathcal{M}_{1}$ and $\mathcal{M}_{2}$ of three modes each.

Table 1. Eigenvalues $\lambda_{c}$ of mass-spring chain.

\begin{tabular}{rrrr}
\hline Mode & Healthy state & Damaged state & Mode set \\
\hline 1 & $-0.255+12.76 \mathrm{i}$ & $-0.253+12.63 \mathrm{i}$ & \\
2 & $-0.747+37.36 \mathrm{i}$ & $-0.736+36.78 \mathrm{i}$ & $\mathcal{M}_{1}$ \\
3 & $-1.16+58.26 \mathrm{i}$ & $-1.15+57.53 \mathrm{i}$ & \\
4 & $-1.55+77.74 \mathrm{i}$ & $-1.54+77.44 \mathrm{i}$ & \\
5 & $-1.96+98.15 \mathrm{i}$ & $-1.96+98.13 \mathrm{i}$ & $\mathcal{M}_{2}$ \\
6 & $-2.13+106.6 \mathrm{i}$ & $-2.11+105.5 \mathrm{i}$ & \\
\hline
\end{tabular}

In the following, localization results at all structural elements are presented for single mode set $\mathcal{M}_{1}$ at one $s$ value, before evaluating the success rate of correct damage localization for single and multiple mode sets at different $s$ values.

Localization results in all elements for mode set $\mathcal{M}_{1}$ at one $s$-value To illustrate the stress computation and its statistical evaluation for damage localization, results for each element of the mass-spring chain are shown in Figure 3 for mode set $\mathcal{M}_{1}$ at one $s$-value. The $s$-value was chosen in the vicinity of mode set $\mathcal{M}_{1}$ as $s=-2+51$ i. Recall that the damage position is inferred by the stress value closest to zero.

In Figure 3(a), the theoretical stress values are computed from the modal parameters corresponding to $\mathcal{M}_{1}$ in the healthy and damaged states. The effect of modal truncation leads to stress that is not exactly zero in damaged element 4 , but that is close to zero and the smallest compared to the stress at the other elements. When computing the stress from modal parameter estimates from simulated datasets in Figure 3(b), the damage position cannot be correctly indicated anymore, which is probably due to variance errors in the estimation from noisy data. Considering the variance of the modal parameters in the method, the damage position is correctly found since the smallest $\chi_{t}^{2}$ value is at element 4 in Figure 3(c).
Success rate of the damage localization using a single mode set After showing the importance of the statistical evaluation for the estimated stress in the damage localization in the last section, we evaluate the success rates of the statistical damage localization based either on single mode set $\mathcal{M}_{1}$ or $\mathcal{M}_{2}$ in dependence of the chosen $s$-value, before going to the joint evaluation of the multiple mode sets in the next section.

For the evaluation of the success rate of correct damage localization at element 4 , using either mode set $\mathcal{M}_{1}$ or $\mathcal{M}_{2}$, 500 datasets of vibration data were generated for the Monte Carlo evaluation. Then, the modes of these datasets and their uncertainties were identified using SSI, both in reference and damaged states. Finally, the success rate was determined based on the computation of the $\chi_{t}^{2}$ test values in (15), using either the modes from $\mathcal{M}_{1}$ or $\mathcal{M}_{2}$, for different $s$-values. The $s$-values were chosen in the vicinity of the modes (see Table 1) on a global grid with $\Re(s) \in[-3,1]$ and $\Im(s) \in[0,130]$.

In Figures 4 and 5, the success rates of correct damage localization (z-axis) are shown in dependence of the real and imaginary part of the chosen $s$-values ( $x$ and $y$-axis) for mode sets $\mathcal{M}_{1}$ and $\mathcal{M}_{2}$, respectively. Indeed it can be seen that damage localization for both mode sets is satisfactory only for $s$-values in the vicinity of the modes of the respective mode sets. For mode set $\mathcal{M}_{1}$, corresponding to the first three modes, Figure 4 shows that the success rate is satisfactory only in the interval of the Laplace variables with $\Im(s) \in[20,64]$, where it reaches up to $90 \%$. Beyond this interval, it is close to zero and the damage position cannot be indicated due to the modal truncation error, which is significant outside the interval containing the identified modes (see Table 1).

Similarly, for mode set $\mathcal{M}_{2}$ corresponding to the last three modes of the structure, it can be seen in Figure 5 that the success rate of damage localization is satisfying with up to $85 \%$ when $\Im(s)$ belongs to the interval $[102,128]$. This area corresponds only to the last two identified modes in $\mathcal{M}_{2}$. While lower performance at $s$-values around the modes of $\mathcal{M}_{1}$ can be expected due to significant modal truncation errors, the success rate at $s$-values near the fourth mode of the structure is also very low. Hence, choosing the $s$-value in the vicinity of the identified poles does not necessarily give perfect results. 


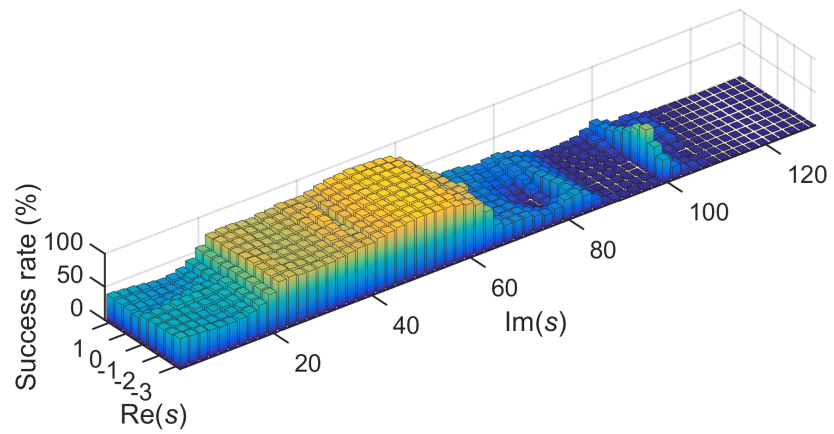

Figure 4. Success rate of statistical damage localization using single mode set $\mathcal{M}_{1}$, in dependence of $s$.

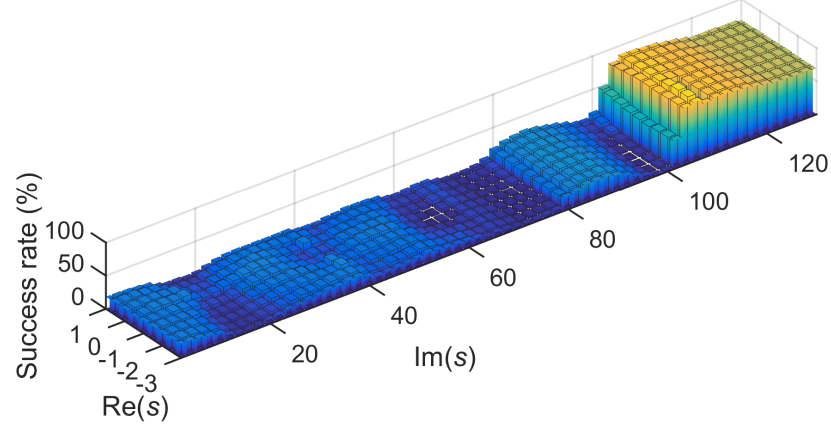

Figure 5. Success rate of statistical damage localization using single mode set $\mathcal{M}_{2}$, in dependence of $s$.

Success rate of the damage localization using multiple mode sets In the previous section, the success rate of the damage localization using a single mode set was not successful everywhere in the $s$-plane mainly because of modal truncation errors. Even considering the $s$-value in the vicinity of the identified modes, where modal truncation errors should be low, was not sufficient to achieve a reasonable success rate for all choices of $s$, especially for $\mathcal{M}_{2}$ in Figure 5. It cannot be known beforehand which $s$ values lead to good results in real experiments, however, the only reasonable assumption is to choose them in the vicinity of the identified modes. This motivates the use of the additional information in multiple mode sets instead of using a single mode set alone, expecting better results for the entire $s$-plane.

For the new statistical approach using multiple mode sets, the $s$-values are chosen separately for each mode set $\mathcal{M}_{1}$ and $\mathcal{M}_{2}$, such that they are in the vicinity of the identified modes of the respective mode set. This means that the $s$-value $s^{1}$ for mode set $\mathcal{M}_{1}$ is chosen with $\Im\left(s^{1}\right) \in[0,64]$, and $s^{2}$ for mode set $\mathcal{M}_{2}$ with $\Im(s) \in[66,128]$, while the real parts are both in the interval $\Re\left(s^{1}\right), \Re\left(s^{2}\right) \in[-3,1]$.

The impact of the choice of $s$-values $s^{1}$ and $s^{2}$ when treating the multiple mode sets has been investigated differently for the respective mode sets. The following two cases are now considered:

Case 1: combination of $\mathcal{M}_{1}$ and $\mathcal{M}_{2}$ with $s$-values

- $s^{1}$ from the entire range in the vicinity of $\mathcal{M}_{1}$, and

- $s^{2}$ with $\Im\left(s^{2}\right)=83$, where it gave a poor performance using $\mathcal{M}_{2}$ only (see Figure 5).

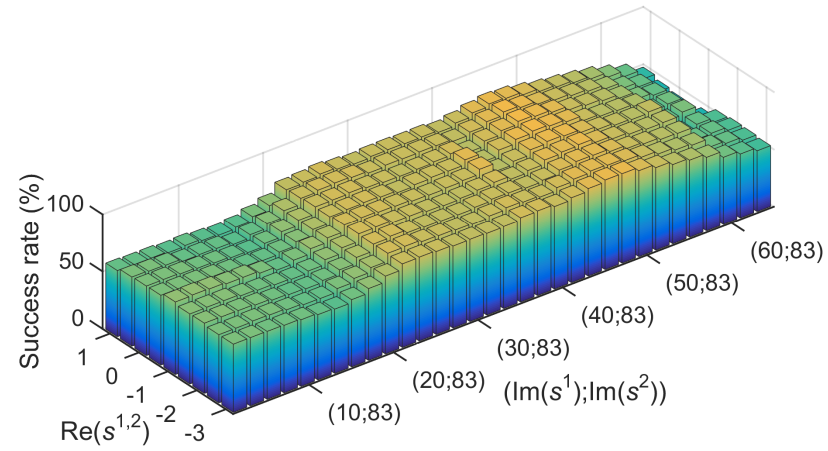

Figure 6. Case 1: success rate of statistical damage localization using multiple mode sets $\mathcal{M}_{1}$ and $\mathcal{M}_{2}$, in dependence of $s^{1}$ and $s^{2}$ with $\Im\left(s^{1}\right) \in[0,64], \Im\left(s_{2}\right)=83$.

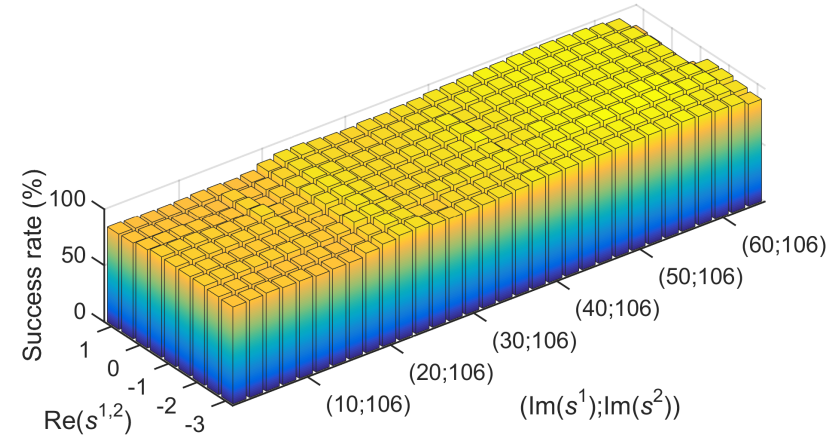

Figure 7. Case 2: success rate of statistical damage localization using multiple mode sets $\mathcal{M}_{1}$ and $\mathcal{M}_{2}$, in dependence of $s^{1}$ and $s^{2}$ with $\Im\left(s^{1}\right) \in[0,64], \Im\left(s_{2}\right)=106$.

In this case, Figure 6 shows that reasonable success rates are achieved in a large range of $s$-values compared to the respective single mode sets, even though the performance for the chosen $s^{2}$ was poor in mode set $\mathcal{M}_{2}$. In the interval $\Im\left(s^{1}\right) \in[20,62]$, the maximum success rate is achieved, which is slightly lower at $80 \%$ than for single mode set $\mathcal{M}_{1}$ in Figure 4, but significantly higher than for single set $\mathcal{M}_{2}$ at the respective $s$-value in Figure 5. In the less optimal region with $\Im\left(s^{1}\right) \in[0,20]$, the success rate using the multiple mode sets is much higher than in the respective regions using the single mode sets. This shows that combining the information from multiple mode sets strongly mitigates the risk of accidentally choosing $s$-values with poor performance in the single mode sets. The overall results with the multiple mode sets are as good or better than the ones in both single mode set cases.

Case 2: combination of $\mathcal{M}_{1}$ and $\mathcal{M}_{2}$ with $s$-values

- $s^{1}$ from the entire range in the vicinity of $\mathcal{M}_{1}$, and

- $s^{2}$ with $\Im\left(s^{2}\right)=106$, where it gave a good performance using $\mathcal{M}_{2}$ only (see Figure 5).

In this case, it can be seen in Figure 7 that the success rate of the damage localization with the new method has significantly improved the situation everywhere in the $s$ plane, compared to all previous results, with success rates between $85 \%$ and $99 \%$.

From both cases 1 and 2 it can be concluded that the treatment of all available modes with the statistical multiple mode set strategy of this paper improves the damage localization performance, compared to the consideration of 
using a limited number of modes in a single mode set in the previous approach ${ }^{16 ; 25 ; 26}$. Choices of the respective $s$-values should be within the vicinity of the respective mode sets. The effect of $s$-values with poor performance are mitigated, and significant improvement on the localization success rate is made through the statistical combination of results in the approach.

\section{Numerical application 2: 3D cube beam model}

In a second numerical application, the damage localization approach has been demonstrated on a 3D cube beam model as shown in Figure 8, considering a more complex structure than in the previous example. The structure is modeled with 9 beam elements of length $10.2 \mathrm{~m}$ (except for the diagonal one). The elements are modeled as pipes with internal and external diameter of $1.08 \mathrm{~m}$ and $1.12 \mathrm{~m}$, respectively. The Young modulus (E), Poisson ratio and mass density of the beam are $210 \mathrm{GPa}, 0.3$ and $7800 \mathrm{~kg} \cdot \mathrm{m}^{-3}$, respectively. The bottom of the elements $1,3,5$ and 7 is fixed to the support. The total number of DOFs of the structure is 24. Damage is introduced in element 8 by decreasing Young and Shear modulus by $50 \%$ of its original value. The evaluated stress resultants for each structural element are the real and imaginary parts of the six internal forces $\tau_{t}=\left\{\begin{array}{ll}F_{x} & M_{x} \\ V_{y} & M_{y} \\ V_{z} & M_{z}\end{array}\right\}$ where $t$ is the element index, $F_{x}$ is the axial force, $V_{y}$ and $V_{z}$ are the shear forces, $M_{x}$ is the moment of torsion and $M_{y}, M_{z}$ the bending moments.

For both healthy and damaged states, acceleration datasets of length $N=200,000$ are simulated from collocated white noise excitation at four accelerometers in the $x-y$-direction at nodes a and d, at a sampling frequency of $350 \mathrm{~Hz}$. White measurement noise with $5 \%$ magnitude of the simulated outputs was added.

In this example, 13 modes can be well identified from the simulated measurements of the structure with SSI. Using four sensors in this example, only a limited set of four modes could be used for localization in previous works ${ }^{16 ; 25 ; 26}$ as the number of modes cannot be bigger than the number of sensors. For localization we consider the first 12 modes, split into three mode sets $\mathcal{M}_{1}, \mathcal{M}_{2}$ and $\mathcal{M}_{3}$ of four modes each for the proposed method in this paper.

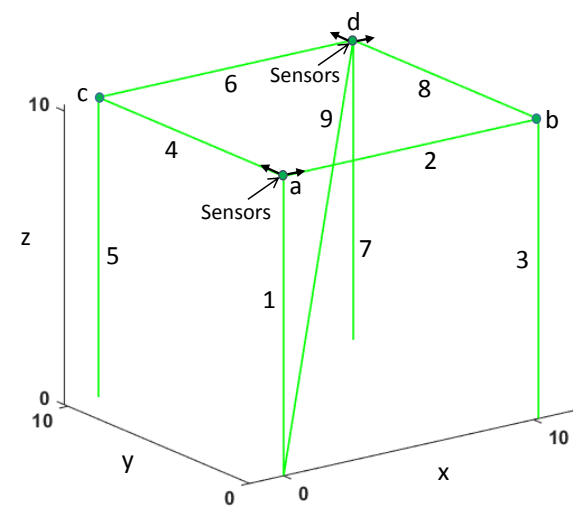

Figure 8. 3D Cube Model with beam elements (24 DOFs)
As in the previous example, we analyze the success rate for localization with the mode sets separately in dependence of the chosen $s$-value, before jointly evaluating it based on all three mode sets with the new method from this paper. Monte Carlo simulations are carried out using 100 simulated datasets in healthy and reference states to determine the success rate, where in each dataset the modes and their uncertainties are identified using SSI. The entire $s$-value range in the vicinity of the modes (see Table 2) is $\Re(s) \in$ $[-12,-7] \cup[-4,-1]$ and $\Im(s) \in[0,650]$.

Table 2. Eigenvalues $\lambda_{c}$ of cube.

\begin{tabular}{rrrr}
\hline Mode & Healthy state & Damaged state & \multicolumn{2}{c}{ Mode set } \\
\hline 1 & $-0.938+46.90 \mathrm{i}$ & $-0.910+45.46 \mathrm{i}$ & \\
2 & $-1.13+56.70 \mathrm{i}$ & $-1.12+56.20 \mathrm{i}$ & \\
3 & $-1.52+76.03 \mathrm{i}$ & $-1.44+71.99 \mathrm{i}$ & $\mathcal{M}_{1}$ \\
4 & $-3.45+172.5 \mathrm{i}$ & $-3.37+169.0 \mathrm{i}$ & \\
5 & $-7.49+374.7 \mathrm{i}$ & $-6.80+340.4 \mathrm{i}$ & \\
6 & $-8.18+409.3 \mathrm{i}$ & $-7.47+373.6 \mathrm{i}$ & \\
7 & $-9.41+470.8 \mathrm{i}$ & $-8.69+435.9 \mathrm{i}$ & \\
8 & $-9.91+495.4 \mathrm{i}$ & $-9.54+477.2 \mathrm{i}$ & \\
9 & $-11.0+552.6 \mathrm{i}$ & $-10.5+527.4 \mathrm{i}$ & \\
10 & $-11.3+566.7 \mathrm{i}$ & $-11.3+564.2 \mathrm{i}$ & \\
11 & $-11.8+591.4 \mathrm{i}$ & $-11.7+586.2 \mathrm{i}$ & $\mathcal{M}_{2}$ \\
12 & $-12.3+615.1 \mathrm{i}$ & $-12.1+606.0 \mathrm{i}$ & \\
& & &
\end{tabular}

In the next section, the success rate of the damage localization results has been computed using the single mode sets separately in Monte-Carlo simulation for 100 datasets. Then, the success rate of the damage localization is illustrated with the new proposed method for the joint statistical evaluation of the multiple mode sets.

Success rate of the damage localization using a single mode set We evaluate the success rates of the statistical damage localization for based either on single mode set $\mathcal{M}_{1}$, $\mathcal{M}_{2}$ or $\mathcal{M}_{3}$ in dependence of the chosen $s$-value, before going to the joint evaluation of the multiple mode sets in the next section.

The $s$-values were chosen within the global range described above to see the influence of the different $s$-values also beyond the range of each individual mode set. In Figures 9, 10 and 11, the success rates of correct damage localization (z-axis) are shown in dependence of the real and imaginary part of the chosen $s$-values ( $x$ and $y$-axis) for mode sets $\mathcal{M}_{1}$, $\mathcal{M}_{2}$ and $\mathcal{M}_{3}$, respectively. Indeed it can be seen that damage localization for both mode sets is satisfactory only for $s$ values in the vicinity of the modes of the respective mode sets.

For mode set $\mathcal{M}_{1}$, corresponding to the first four modes in the region $\Im(s) \in[0,175]$, Figure 9 shows that the success rate is satisfactory only in the interval of the Laplace variables with $\Im(s) \in[0,81] \cup[120,201]$, where it reaches up to $100 \%$. Beyond this interval, it is close to zero and the damage position cannot be indicated due to the modal truncation error, which is significant outside the interval containing the identified modes (see Table 1). However, there is also a region within the vicinity of the modes of $\mathcal{M}_{1}$, where the success rate is close to zero.

The four modes in mode set $\mathcal{M}_{2}$ are within the region $\Im(s) \in[300,475]$, and it can be seen in Figure 10 that the success rate of damage localization is satisfying in the 


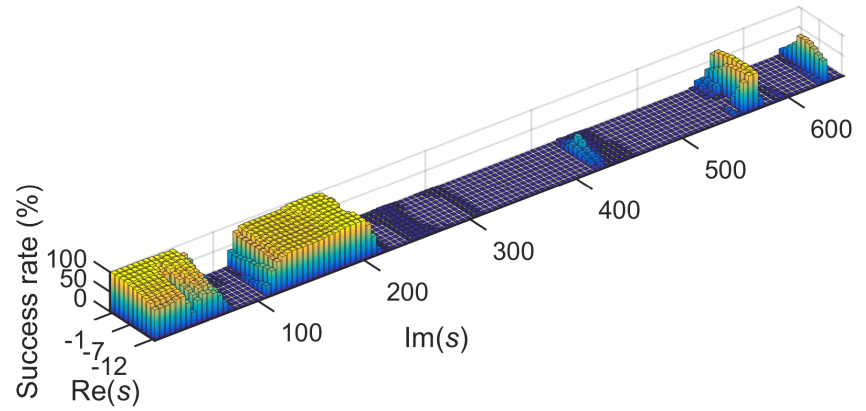

Figure 9. Success rate of statistical damage localization using single mode set $\mathcal{M}_{1}$, in dependence of $s$.

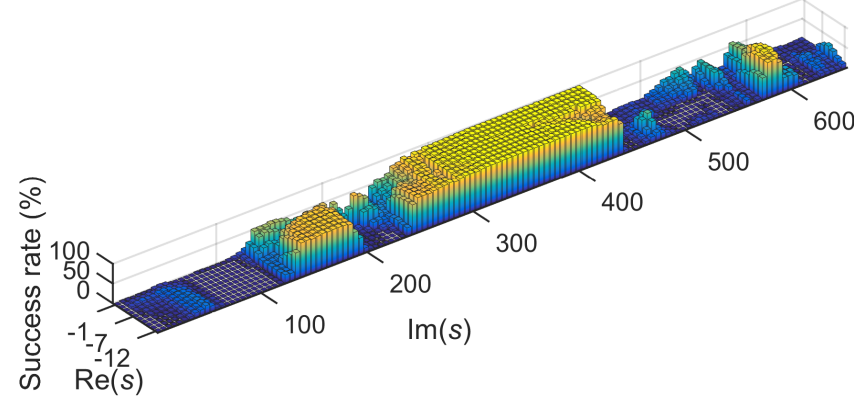

Figure 10. Success rate of statistical damage localization using single mode set $\mathcal{M}_{2}$, in dependence of $s$.

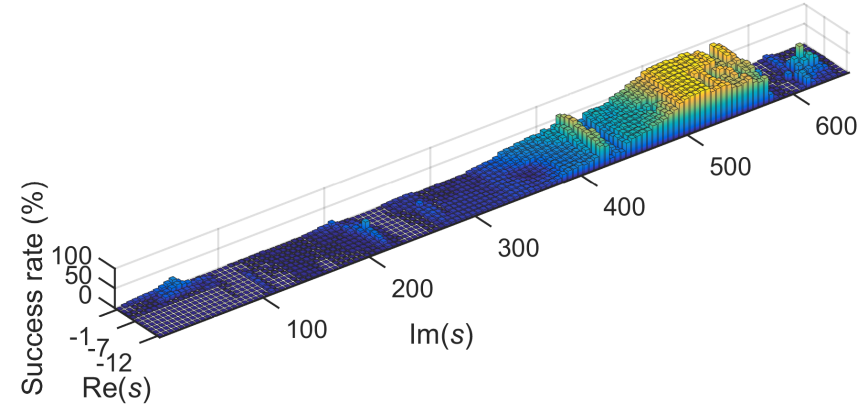

Figure 11. Success rate of statistical damage localization using single mode set $\mathcal{M}_{3}$, in dependence of $s$.

interval $\Im(s) \in[300,445]$, covering nearly the entire region. Possibly, the success rate in the last part of the region is low due to proximity of mode set $\mathcal{M}_{3}$ and the resulting truncation errors.

Finally, the success rate for mode set $\mathcal{M}_{3}$ is shown in Figure 11, where the modes are in the interval $\Im(s) \in$ $[450,625]$. The success rate is $\operatorname{good}$ for $\Im(s) \in[500,580]$, covering only a part of the region corresponding to $\mathcal{M}_{3}$.

Similarly as in the previous example, this shows that choosing the $s$-value in the vicinity of the identified poles does not necessarily give perfect results. This motivates the combination of results of different mode sets for more robustness and less dependence on the particular choice of $s$.

Success rate of the damage localization using multiple mode sets For the new statistical approach using multiple mode sets, the $s$-values are chosen separately for each mode set $\mathcal{M}_{1}, \mathcal{M}_{2}$ and $\mathcal{M}_{3}$, such that they are in the vicinity of the identified modes of the respective mode set. Therefore, the range of the respective $s$-values $s^{1}, s^{2}$ and $s^{3}$

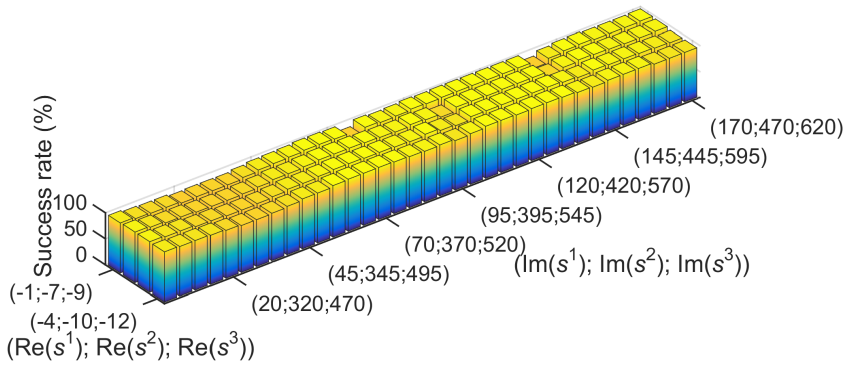

Figure 12. Success rate of statistical damage localization using multiple mode sets $\mathcal{M}_{1}, \mathcal{M}_{2}$ and $\mathcal{M}_{3}$, in dependence of $s^{1}, s^{2}$ and $s^{3}$.

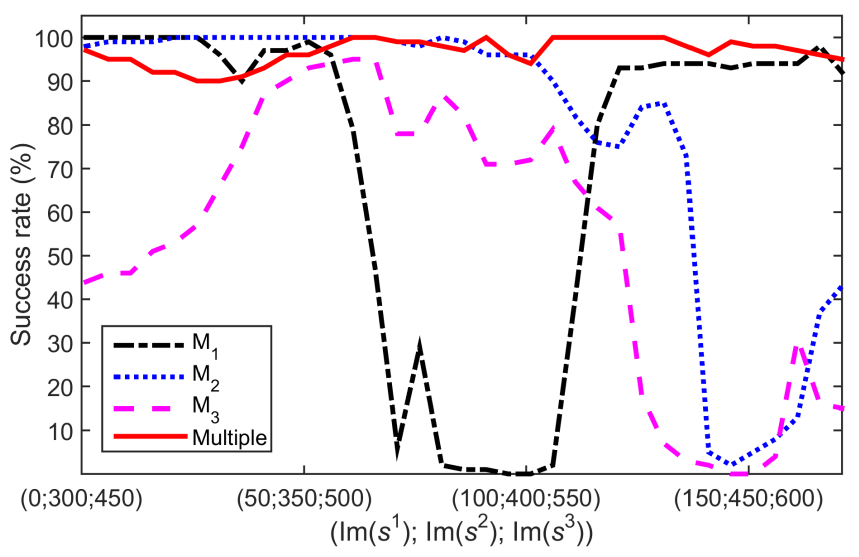

Figure 13. Success rate of statistical damage localization in the single mode sets $\mathcal{M}_{1}, \mathcal{M}_{2}$ and $\mathcal{M}_{3}$ with $s$ in the vicinity of the respective modes, compared to the success rate using jointly the multiple mode sets.

is chosen with $\Re\left(s^{1}\right) \in[-4,-1], \Im\left(s^{1}\right) \in[0,175], \Re\left(s^{2}\right) \in$ $[-10,-7], \Im\left(s^{2}\right) \in[300,475], \Re\left(s^{3}\right) \in[-12,-9], \Im\left(s^{3}\right) \in$ $[450,625]$.

The impact of the choice of $s$-values $s^{1}, s^{2}$ and $s^{3}$ in the respective mode sets has been investigated for the joint statistical evaluation of the stress, where the evaluation for each triplet $\left(s^{1}, s^{2}, s^{3}\right)$ is carried out with $s^{1}, s^{2}$ and $s^{3}$ covering the above range of $s$-values used in the respective mode sets. In Figure 12, the resulting success rate of the joint evaluation is shown, where it can be seen that the success rate is nearly at $100 \%$ everywhere in the $s$-plane. A comparison to the success rates from the single mode sets in the respective range of $s$-values is made in Figure 13 for fixed real parts $\Re\left(s^{1}\right)=-3, \Re\left(s^{2}\right)=-9$ and $\Re\left(s^{3}\right)=-11$, where it can be clearly seen that the statistical combination of the results from the single mode sets significantly improves the damage localization performance nearly everywhere in the $s$-plane. In particular, the choice of $s$-values in the vicinity of the identified modes always yields very good success rates in the joint evaluation of the multiple mode sets, while this was not always the case using the single mode sets separately.

\section{Experimental application: cantilever beam}

Experimental setup and measurements In a lab experiment, damage tests were conducted on a cantilever beam that is made of plastic, as shown in Figure 14. Its dimensions are $0.5 \mathrm{~m} \times 0.08 \mathrm{~m} \times 0.01 \mathrm{~m}$ and it is fixed on one side. Damage 


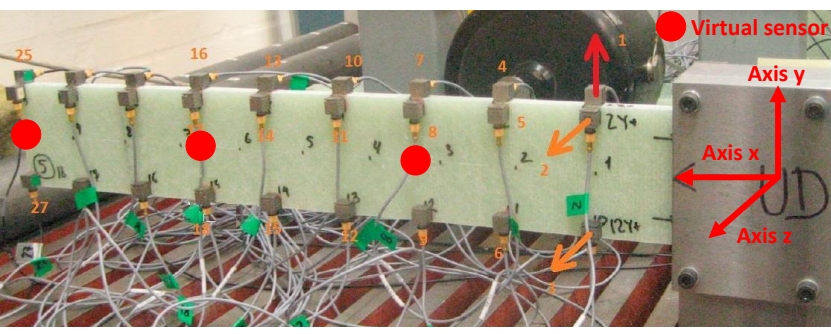

Figure 14. Experimental setup of the beam.

was introduced by drilling holes, located at $0.08 \mathrm{~m}$ from the fixed end. The beam was excited horizontally by a shaker under white noise excitation and the response was monitored by 18 horizontal and 9 vertical accelerometers. For both the healthy and damaged states, acceleration data of length $N=295,936$ with a sampling frequency of $8192 \mathrm{~Hz}$ were recorded.

This experimental setup has been used previously as a validation case for the SDDLV method ${ }^{26}$. In this previous work, the localization was performed using all the available horizontal sensors. Consequently, using all the signals it was possible to identify natural frequencies and modes shape in bending and torsion.

The objective of the present study is to localize the damage with a minimum number of sensors to highlight the advantage of using the proposed multiple mode sets method. Furthermore, it is intended to demonstrate the method based on a very simple finite element model of the structure.

Therefore, we consider only three "virtual sensors" located in the center of the structure instead of the full data from the horizontal accelerometers (see Figure 14). The measurements of these three sensors at $0.167 \mathrm{~m}, 0.333 \mathrm{~m}$ and $0.5 \mathrm{~m}$ from the fixed end are obtained as the mean of the measured accelerations at the top and bottom of the beam. This means that we are voluntarily excluding the torsional modes. We are able to identify five bending modes in the plane $x-z$, which will show to be sufficient for a precise damage localization in the following when considering all five modes in two mode sets with the method from this paper.

Modal analysis and uncertainties After downsampling and decimation of the data by factor 4 , five well-estimated bending modes were obtained in the healthy and the three damaged states from the measurement data using SSI, together with their uncertainties. The identified frequencies are shown in Table 3 for each mode. Since only three sensors are used, the identified modes are split into two mode sets $\mathcal{M}_{1}$ and $\mathcal{M}_{1}$ containing two and three modes, respectively.

Table 3. Identified frequencies (in $\mathrm{Hz}$ ) of beam.

\begin{tabular}{rrrrrr}
\hline Mode & healthy & 1 hole & 3 holes & 5 holes & Mode set \\
\hline 1 & 16.53 & 16.41 & 16.20 & 15.83 & \\
2 & 81.06 & 81.44 & 81.52 & 81.47 & \multirow{2}{*}{$\mathcal{M}_{1}$} \\
3 & 326.9 & 322.9 & 326.3 & 327.2 & \\
4 & 529.4 & 528.4 & 527.1 & 523.9 & \\
5 & 831.3 & 831.8 & 831.5 & 826.1 & $\mathcal{M}_{2}$ \\
\hline
\end{tabular}

Finite element model The model needs to be as simple as is practicable but it has to correctly represent the behavior of

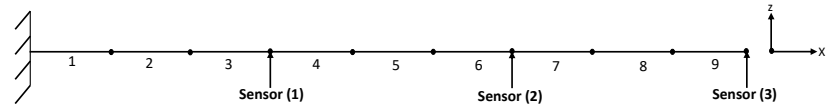

Figure 15. Model of the beam with 3 sensors.

the structure. In this particular example, a simple 2D beam model gives the correct mode shapes for the bending modes in the plane $x-z$, so it can represent the first five bending modes in the plane $x-z$ of the structure. Considering all the modes, it matches to the modes 1-2-5-6-9 of a 3D model used as reference. Here, the structure is not strictly speaking a beam since the ratio length/width is inferior to 10 . This leads to a systematic, but acceptable, model error of about $8 \%$ considering the gap between the reference values of the eigenfrequencies obtained with a very fine mesh $3 \mathrm{D}$ model and the ones obtained with the analytical beam model.

A trade-off between model size, i.e. the number of DOFs, and model precision must be considered for the computation of the stress for damage localization. The stress resultants and their uncertainties are computed for each structural element. Using only three sensors it seems to be reasonable to demand a localization precision within a discretization of nine elements of the beam. Additionally, such a coarse mesh of nine elements is good enough to compute the first five bending modes in the plane $x-z$. It is finally the model that we have chosen in this study, as depicted in Figure 15.

The Young modulus (E), Poisson ratio and mass density of the beam are chosen as $5 \mathrm{GPa}, 0.4$ and $1100 \mathrm{~kg} . \mathrm{m}^{-3}$, respectively. The computed stress resultants for each structural element are the real and imaginary parts of the three internal forces $\tau_{t}=\left\{\begin{array}{cc}F_{x} & 0 \\ 0 & M_{y} \\ V_{z} & 0\end{array}\right\}$. The damage is situated at element 2 in this model, being located at $0.08 \mathrm{~m}$ from the fixed end.

Localization results at all elements The performance of the proposed damage localization method using multiple mode sets is compared to the separate single mode set evaluation for the three different damage scenarios.

The localization results are computed at all elements from the experimental datasets in both damaged and healthy states. The computation of the stress and its uncertainties for the statistical evaluation in the $\chi_{t}^{2}$-tests is carried out for two different sets of $s$-values, each in the vicinity of the respective mode sets. First, one $s$-value is chosen for each mode set with $s^{1}=-10+90$ i for mode set $\mathcal{M}_{1}$ and $s^{2}=-10+3200 \mathrm{i}$ for mode set $\mathcal{M}_{2}$. Second, three $s$-values are chosen for joint evaluation for each mode set as in (16) (17): $s_{1}^{1}=-10+90 \mathrm{i}, s_{2}^{1}=-10+100 \mathrm{i}, s_{3}^{1}=-10+200 \mathrm{i}$ for $\mathcal{M}_{1}$, and $s_{1}^{2}=-10+2100 \mathrm{i}, s_{2}^{2}=-10+3200 \mathrm{i}, s_{3}^{2}=$ $-10+3300 \mathrm{i}$ for $\mathcal{M}_{2}$. To easily compare the ratios of the stress or associated $\chi_{t}^{2}$-values between the undamaged and damaged elements in the different test cases, the computed values are normalized in the figures such that the smallest value of the nine elements is 1 .

The estimated stress and its statistical evaluation is shown in detail for the first damage case of 1 hole. In Figures 16 and 17 , the results are shown for considering the single mode sets 


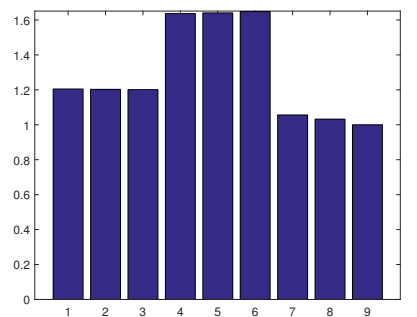

(a) Estimated stress

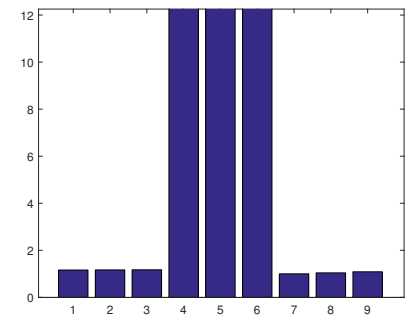

(b) Statistical evaluation in $\chi_{t}^{2}$-tests
Figure 16. Localization results for all elements using single mode set $\mathcal{M}_{1}$ at $s^{1}=-10+90$ i. Damage case: 1 hole.

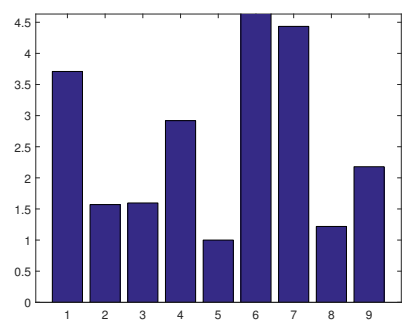

(a) Estimated stress

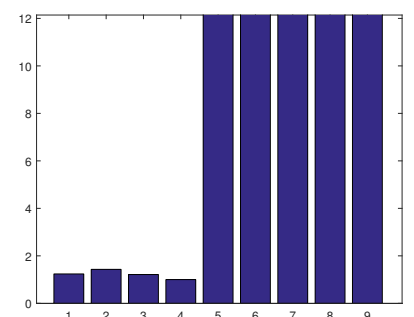

(b) Statistical evaluation of $\chi_{t}^{2}$-tests
Figure 17. Localization results for all elements using single mode set $\mathcal{M}_{2}$ at $s^{2}=-10+3200$ i. Damage case: 1 hole.

$\mathcal{M}_{1}$ and $\mathcal{M}_{2}$. Neither the estimated stress nor its statistical evaluation can correctly indicate the damage at element 2 . When using the joint statistical evaluation of both mode sets with the method of this paper, the results using one $s$ value in Figure 18(a) indicate the damaged element within the adjacent elements of the damaged one, being close to correct damage localization. By adding information through two more $s$-values in the same setting, the damaged element can be correctly indicated in Figure 18(b).

Then, the localization results with the proposed method of this paper are presented in Figures 19 and 20 for the further damage severities of three and five holes, showing the joint statistical evaluation of the multiple mode sets using one or three $s$-values for each mode set. Using one $s$-value in Figures 19(a) and 20(a), the damage can be localized in the region of the damaged and its adjacent elements, and the $\chi_{t^{-}}^{2}$ value for the damaged element 2 is slightly lower than for the neighboring elements. Using three $s$-values in Figures 19(b) and 20(b), the damage can be more clearly localized in element 2.

Summarizing the results in this experiment, it can be seen that neither the estimated stress from the SDDLV approach nor its statistical evaluation is sufficient for damage localization in the classical formulation using one mode set, which is probably due to significant modal truncation errors when using only limited modal information. The statistical evaluation with the proposed method takes all the modal information into account and correctly indicated the damage locations in this example, based on measurements from only three sensors and five identified modes, together with a coarse FE model of the structure.

\section{Conclusions}

In this paper, the damage localization with the SDDLV approach has been extended considering multiple mode sets

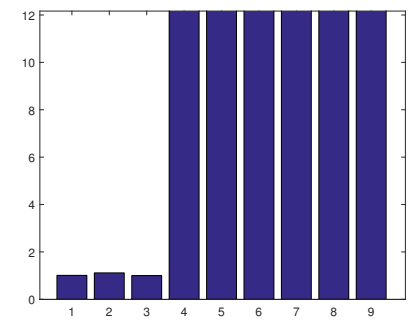

(a) $1 s$-value

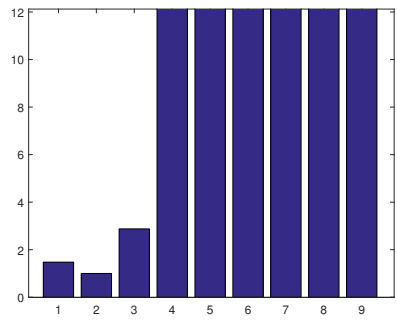

(b) $3 s$-values
Figure 18. Statistical localization results with new method using both mode sets. Damage case: 1 hole.

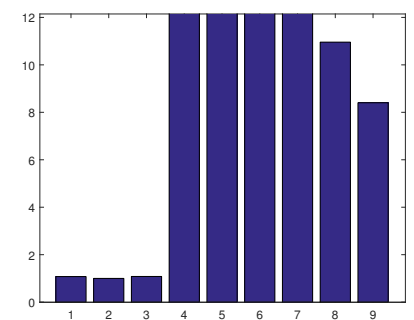

(a) $1 s$-value

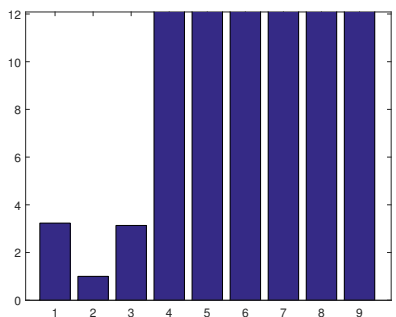

(b) $3 s$-values
Figure 19. Statistical localization results with new method using both mode sets. Damage case: 3 holes.

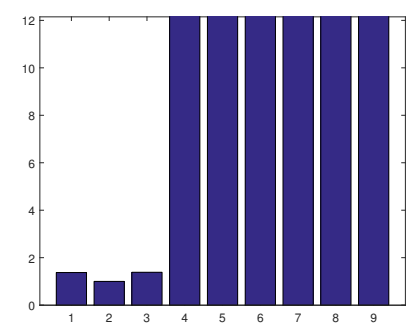

(a) $1 s$-value

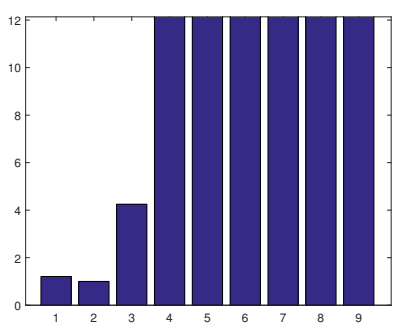

(b) $3 s$-values
Figure 20. Statistical localization results with new method using both mode sets. Damage case: 5 holes.

based on a joint statistical evaluation that takes into account the information from all identified modes of the structure. The stress computation using multiple mode sets increases the information content about the damaged or non-damaged elements of the structure, compared to evaluation from a limited number of modes due to a previous constraint of the approach on the number of modes. With the new approach, this constraint is lifted, which allows damage localization with fewer sensors at the same time. While the stress evaluation for each mode set is naturally subject to modal truncation errors that depend on the choice of the Laplace variable $s$, the joint statistical evaluation for several mode sets seems to mitigate these errors. Indeed, the simulation results in the numerical applications show that the effect of $s$-values with poor performance in single mode sets is mitigated when treating all available modes with the statistical multiple mode set strategy of this paper. This leads to significant improvement of the localization success rate with the proposed method and less dependence on the particular choice of the $s$-values, which contributes to the applicability of the method in practice in SHM systems. Finally, the proposed method was able to correctly localize the damage in an experimental application on a damaged 
cantilever beam with a small number of sensors and using a coarse FE model. Future works should include a detailed analysis of the performance of the localization method under environmental nuisances like temperature changes in combination with methods that remove the environmental variability on modal parameters, in order to further improve the applicability on structures in the field.

\section{Acknowledgements}

Financial support from the Bretagne region for the first author is gratefully acknowledged. Furthermore, we thank Brüel \& Kjær for providing the data from the beam experiment.

\section{Appendix A. Stochastic subspace identification}

From discrete measurements of system (2), the modal parameters are identified based on covariance-driven stochastic subspace identification. The corresponding discrete-time state-space model is

$$
\left\{\begin{aligned}
x_{k+1} & =A_{d} x_{k}+v_{k} \\
y_{k} & =C_{d} x_{k}+w_{k}
\end{aligned}\right.
$$

where $A_{d}=\exp \left(A_{c} \tau\right), C_{d}=C_{c}, \tau$ is the time step, $v_{k}$ and $w_{k}$ are process and measurement noise, respectively. Using covariance-driven $\mathrm{SSI}^{29 ; 30}$, estimates of the system matrices $\widehat{A}_{d}$ and $\widehat{C}_{d}$ are identified at different model orders from the measurements. Using noisy data, these model orders require to be relatively high and additionally noise modes appear in the results. From these results the modes are selected in a so-called stabilization diagram ${ }^{30}$. After rejecting the noise modes, finally the corresponding eigenvalues of the continuous-time system and the mode shapes are used to get the desired estimates $\widehat{A}_{c}$ and $\widehat{C}_{c}$.

\section{A1: System identification}

A sensor subset of so-called reference sensors or projection channels can be used to reduce the computational burden and to improve identification ${ }^{29}$, denoted by $y_{k}^{(\mathrm{ref})}$. Let the theoretical cross-covariance between the state and the reference outputs be $\mathcal{G}=\mathbf{E}\left(x_{k+1} y_{k}^{(\mathrm{ref}) T}\right)$ and the output covariance be $\mathcal{R}_{i}=\mathbf{E}\left(y_{k} y_{k-i}^{(\mathrm{ref}) T}\right)=C_{d} A_{d}^{i-1} \mathcal{G}$, then the block Hankel matrix is formulated as

$$
\mathcal{H}=\left[\begin{array}{cccc}
\mathcal{R}_{1} & \mathcal{R}_{2} & \ldots & \mathcal{R}_{q} \\
\mathcal{R}_{2} & \mathcal{R}_{3} & \ldots & \mathcal{R}_{q+1} \\
\vdots & \vdots & \ddots & \vdots \\
\mathcal{R}_{p+1} & \mathcal{R}_{p+2} & \ldots & \mathcal{R}_{p+q}
\end{array}\right]=\operatorname{Hank}\left(\mathcal{R}_{i}\right)
$$

It has the factorization property $\mathcal{H}=\mathcal{O C}$ into the matrices of observability and controllability

$$
\mathcal{O}=\left[\begin{array}{c}
C_{d} \\
C_{d} A_{d} \\
\vdots \\
C_{d} A_{d}^{p}
\end{array}\right] \text { and } \mathcal{C}=\left[\begin{array}{llll}
\mathcal{G} & A_{d} \mathcal{G} & \ldots & A_{d}^{q-1} \mathcal{G}
\end{array}\right]
$$

respectively. From $\mathcal{O}$, the observation matrix $C_{d}$ is obtained in its first block row. Using the shift-invariance property of $\mathcal{O}$, the matrix $A_{d}$ is obtained based on least squares solution $A_{d}=\mathcal{O}^{\uparrow} \mathcal{O}^{\downarrow}$, where the last and the first block row has been eliminated in $\mathcal{O}^{\uparrow}$ and $\mathcal{O}^{\downarrow}$.

Using measurement data, the output covariance estimates $\widehat{\mathcal{R}}_{i}=1 / N \sum_{k=1}^{N} y_{k} y_{k-i}^{(\mathrm{ref}) T}, i=1, \ldots, p+q$, are computed to get the estimation of the Hankel matrix $\widehat{\mathcal{H}}=\operatorname{Hank}\left(\widehat{\mathcal{R}}_{i}\right)$ in (18). From an SVD of $\widehat{\mathcal{H}}$, the observability matrix $\widehat{\mathcal{O}}$ is estimated and the system matrices $\widehat{A}_{d}$ and $\widehat{C}_{d}$ are obtained as above.

\section{A2: Modes}

For the discrete-time system, the eigenvalues $\lambda_{d, l}$ and mode shapes $\varphi_{l}$ are obtained from the system matrices $A_{d}$ and $C_{d}$ for each mode $l$ as

$$
\operatorname{det}\left(A_{d}-\lambda_{d, l} I\right)=0, \quad A_{d} \phi_{l}=\lambda_{d, l} \phi_{l}, \quad \varphi_{l}=C_{d} \phi_{l} .
$$

The respective eigenvalues of system matrix $A_{c}$ in the continuous-time system are retrieved as

$$
\lambda_{c, l}=\frac{1}{\tau} \log \left(\lambda_{d, l}\right)
$$

and the natural frequencies $f_{l}$ and damping ratios $\xi_{l}$ yield

$$
f_{l}=\frac{\left|\lambda_{c, l}\right|}{2 \pi \tau}, \quad \xi_{l}=\frac{-\Re\left(\lambda_{c, l}\right)}{\left|\lambda_{c, l}\right|} .
$$

When estimating the modal parameters for different system orders, the structural modes can be distinguished from spurious noise modes in the well-known stabilization diagram procedure.

\section{A3: Final system matrices}

Let $m$ be the number of mode pairs selected from the stabilization diagram, satisfying $m \leq r$. Let the identified modes be $\left(\lambda_{c, l}, \varphi_{l}\right)$ and their conjugated complex $\left(\overline{\lambda_{c, l}}, \bar{\varphi}_{l}\right)$, $l=1, \ldots, m$. Then, the system matrices in the real-valued modal basis can be written as ${ }^{26}$

$$
A_{c}=\left[\begin{array}{cc}
\Re\left(\Lambda_{c}\right) & \Im\left(\Lambda_{c}\right) \\
-\Im\left(\Lambda_{c}\right) & \Re\left(\Lambda_{c}\right)
\end{array}\right], \quad C_{c}=[\Re(\Phi) \quad \Im(\Phi)],
$$

where $\Lambda_{c}=\operatorname{diag}\left(\lambda_{c}^{1}, \ldots, \lambda_{c}^{m}\right)$ and $\Phi=\left[\begin{array}{lll}\varphi^{1} & \ldots & \varphi^{m}\end{array}\right]$, obtained from the chosen modes.

\section{Appendix B. Covariance of Hankel matrix}

The covariance $\hat{\Sigma}_{\mathcal{H}}=\operatorname{cov}(\operatorname{vec}(\mathcal{H}))$ of the Hankel matrix can be directly computed by separating the available measurement data into $n_{b}$ blocks of length $N_{b}{ }^{20 ; 22}$. The Hankel matrix $\hat{\mathcal{H}}^{(k)}=\operatorname{Hank}\left(\hat{\mathcal{R}}_{i}^{(k)}\right)$ is computed from the output covariances $\hat{\mathcal{R}}_{i}^{(k)}=1 / N_{b} \sum_{k=1+(k-1) N_{b}}^{k N_{b}} y_{k} y_{k-i}^{(\text {ref }) T}$ on each block, while the Hankel matrix on the entire dataset yields $\hat{\mathcal{H}}=\frac{1}{n_{b}} \sum_{k=1}^{n_{b}} \hat{\mathcal{H}}^{(k)}$. Finally, the covariance $\hat{\Sigma}_{\mathcal{H}}$ is estimated by the sample covariance

$$
\hat{\Sigma}_{H}=\frac{1}{n_{b}-1} \sum_{k=1}^{n_{b}} \operatorname{vec}\left(\hat{\mathcal{H}}^{(k)}-\hat{\mathcal{H}}\right) \operatorname{vec}\left(\hat{\mathcal{H}}^{(k)}-\hat{\mathcal{H}}\right)^{T} .
$$




\section{References}

1. Farrar C, Doebling S and Nix D. Vibration-based structural damage identification. Philosophical Transactions of the Royal Society A: Mathematical, Physical and Engineering Science 2001; 359(1778): 131-149.

2. Carden E and Fanning P. Vibration based condition monitoring: a review. Structural Health Monitoring 2004; 3(4): 355-377.

3. Fan W and Qiao P. Vibration-based damage identification methods: a review and comparative study. Structural Health Monitoring 2011; 10(1): 83-111.

4. Farrar C and Worden K. An introduction to structural health monitoring. Philosophical Transactions of the Royal Society A: Mathematical, Physical and Engineering Sciences 2007; 365(1851): 303-315.

5. Deraemaeker A, Reynders E, De Roeck G et al. Vibrationbased structural health monitoring using output-only measurements under changing environment. Mechanical Systems and Signal Processing 2008; 22(1): 34-56.

6. Balmès E, Basseville $\mathrm{M}$, Bourquin $\mathrm{F}$ et al. Merging sensor data from multiple temperature scenarios for vibration-based monitoring of civil structures. Structural Health Monitoring 2008; 7(2): 129-142.

7. Döhler M, Mevel L and Hille F. Subspace-based damage detection under changes in the ambient excitation statistics. Mechanical Systems and Signal Processing 2014; 45(1): $207-$ 224.

8. Döhler M, Hille F, Mevel L et al. Structural health monitoring with statistical methods during progressive damage test of S101 Bridge. Engineering Structures 2014; 69: 183-193.

9. Comanducci G, Magalhães F, Ubertini F et al. On vibrationbased damage detection by multivariate statistical techniques: Application to a long-span arch bridge. Structural Health Monitoring 2016; 15(5): 505-524.

10. Brownjohn JMW, Xia PQ, Hao $\mathrm{H}$ et al. Civil structure condition assessment by FE model updating: methodology and case studies. Finite Elements in Analysis and Design 2001; 37(10): 761-775.

11. Simoen E, De Roeck G and Lombaert G. Dealing with uncertainty in model updating for damage assessment: A review. Mechanical Systems and Signal Processing 2015; 56: 123-149.

12. Limongelli M, Chatzi E, Döhler M et al. Towards extraction of vibration-based damage indicators. In Proc. 8th European Workshop on Structural Health Monitoring. Bilbao, Spain.

13. Balmès E, Basseville M, Mevel L et al. Statistical modelbased damage localization: a combined subspace-based and substructuring approach. Structural Control and Health Monitoring 2008; 15(6): 857-875.

14. Döhler M, Mevel L and Zhang Q. Fault detection, isolation and quantification from Gaussian residuals with application to structural damage diagnosis. Annual Reviews in Control 2016; 42: 244-256.

15. Bernal D. Load vectors for damage localization. Journal of Engineering Mechanics 2002; 128(1): 7-14.

16. Bernal D. Load vectors for damage location in systems identified from operational loads. Journal of Engineering Mechanics 2010; 136(1): 31-39.

17. Mazars J. A description of micro-and macroscale damage of concrete structures. Engineering Fracture Mechanics 1986; 25(5-6): 729-737.
18. Zheng DY and Kessissoglou NJ. Free vibration analysis of a cracked beam by finite element method. Journal of Sound and Vibration 2004; 273(3): 457-475.

19. Pintelon R, Guillaume P and Schoukens J. Uncertainty calculation in (operational) modal analysis. Mechanical Systems and Signal Processing 2007; 21(6): 2359-2373.

20. Reynders E, Pintelon R and De Roeck G. Uncertainty bounds on modal parameters obtained from stochastic subspace identification. Mechanical Systems and Signal Processing 2008; 22(4): 948-969.

21. Carden E and Mita A. Challenges in developing confidence intervals on modal parameters estimated for large civil infrastructure with stochastic subspace identification. Structural Control and Health Monitoring 2011; 18(1): 53-78.

22. Döhler M and Mevel L. Efficient multi-order uncertainty computation for stochastic subspace identification. Mechanical Systems and Signal Processing 2013; 38(2): 346-366.

23. Döhler M, Lam XB and Mevel L. Uncertainty quantification for modal parameters from stochastic subspace identification on multi-setup measurements. Mechanical Systems and Signal Processing 2013; 36(2): 562-581.

24. Mellinger P, Döhler M and Mevel L. Variance estimation of modal parameters from output-only and input/output subspacebased system identification. Journal of Sound and Vibration 2016; 379: 1-27.

25. Döhler M, Marin L, Bernal D et al. Statistical decision making for damage localization with stochastic load vectors. Mechanical Systems and Signal Processing 2013; 39(1-2): 426-440.

26. Marin L, Döhler M, Bernal D et al. Robust statistical damage localization with stochastic load vectors. Structural Control and Health Monitoring 2015; 22(3): 557-573.

27. Bernal D. Flexibility-based damage localization from stochastic realization results. Journal of Engineering Mechanics 2006; 132(6): 651-658.

28. Van Overschee P and De Moor B. Subspace Identification for Linear Systems: Theory, Implementation, Applications. Kluwer, 1996.

29. Peeters B and De Roeck G. Reference-based stochastic subspace identification for output-only modal analysis. Mechanical Systems and Signal Processing 1999; 13(6): 855878.

30. Döhler $M$ and Mevel L. Fast multi-order computation of system matrices in subspace-based system identification. Control Engineering Practice 2012; 20(9): 882-894.

31. Sohn H. Effects of environmental and operational variability on structural health monitoring. Philosophical Transactions of the Royal Society A: Mathematical, Physical and Engineering Sciences 2007; 365(1851): 539-560.

32. Magalhães F, Cunha A and Caetano E. Vibration based structural health monitoring of an arch bridge: From automated OMA to damage detection. Mechanical Systems and Signal Processing 2012; 28: 212-228.

33. Hannan E. Multiple time series. New York: Wiley, 1970.

34. Casella G and Berger R. Statistical inference. Pacific Grove, CA, USA: Duxbury Press, 2002. 